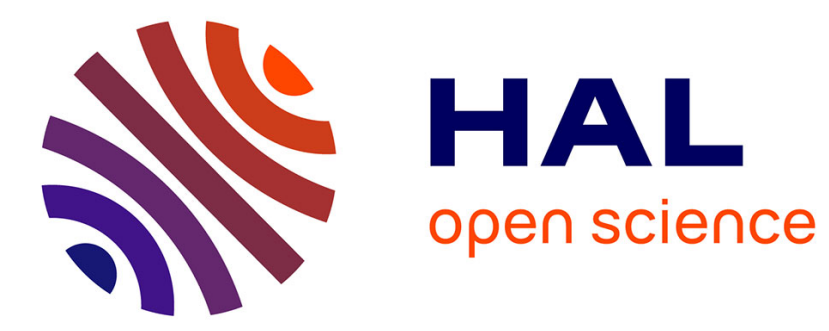

\title{
Stabilization of polytopes of plants by their vertices
}

Christophe Fonte, Houda Meddeb, Michel Zasadzinski

\section{To cite this version:}

Christophe Fonte, Houda Meddeb, Michel Zasadzinski. Stabilization of polytopes of plants by their vertices. International Journal of Robust and Nonlinear Control, 2019, 29 (18), pp.6398-6418. 10.1002/rnc.4731 . hal-02355289v2

\section{HAL Id: hal-02355289 \\ https://hal.science/hal-02355289v2}

Submitted on 20 Dec 2019

HAL is a multi-disciplinary open access archive for the deposit and dissemination of scientific research documents, whether they are published or not. The documents may come from teaching and research institutions in France or abroad, or from public or private research centers.
L'archive ouverte pluridisciplinaire HAL, est destinée au dépôt et à la diffusion de documents scientifiques de niveau recherche, publiés ou non, émanant des établissements d'enseignement et de recherche français ou étrangers, des laboratoires publics ou privés. 


\title{
Stabilization of polytopes of plants by their vertices
}

\author{
Christophe Fonte, Houda Meddeb and Michel Zasadzinski \\ Centre de Recherche en Automatique de Nancy, CRAN, UMR 7039, \\ Université de Lorraine, 186 rue de Lorraine, 54400 Cosnes et Romain, France and CNRS. \\ Email: $\{$ christophe.fonte,houda.meddeb,michel.zasadzinski\}@univ-lorraine.fr.
}

\begin{abstract}
This paper aims to assess the stability of a polytope of linear systems by their vertices. These results are based on the Hermite-Biehler and Edge theorems. A sufficient condition satisfying a constraint on the even (or odd) part of the closed-loop characteristic polynomials associated with the stabilization of its vertices, is proved. Finally, a constructive method to stabilize a polytope of plants with simultaneous and robust linear time-invariant controllers is established.
\end{abstract}

Keywords: polytope of systems, simultaneous stabilization, robust stabilization, common interlacing property.

\section{Introduction}

This paper concerns the stabilization of polytopes of SISO plants with a fixed order controller. Until now the problem of deciding of the stability or stabilizability of a polytope of linear systems with a linear time-invariant (LTI) controller stays an open question. We do not know which are the conditions so that any polytope of systems is stabilizable by a LTI controller. In this article, we show that this question is equivalent to that of the stability of the set of segments of systems linking the vertices of the polytope with a LTI controller. The issue is therefore about the stability of this set of segments with a LTI controller. Remark that this problem is also an open question. This is even an undecidable mathematical issue that may be classified as an NP-hard problem, in the sense that it is not possible to find necessary and sufficient conditions to stabilize this family of systems with a LTI controller when the number of plants to stabilize, is greater than two, see [1]. Accordingly, there exist only sufficient or necessary conditions for the robust stabilization of a polytope of systems. This paper proposes some sufficient conditions based on the Edge theorem [2]. More precisely, the question of stabilization of a polytope of systems is treated as a simple simultaneous control design of a family of LTI systems submitted to an additional constraint on the closed-loop characteristic polynomials associated with the vertices of this polytope. Furthermore, the controller is shown to not only simultaneously stabilize the vertices of the polytope of systems, i.e. a simultaneous control purpose, but also the set of systems belonging to the polytope, i.e. a robust control purpose. That is hence a simultaneous and robust controller for the polytopes of systems. Let us review briefly the main results in simultaneous and robust control.

Concerning the simultaneous control, [3] and [4] have proved that there exits a necessary and sufficient condition to stabilize simultaneously two LTI systems. Moreover, [3] and [4] have shown 
that this question is equivalent to the stabilization of one plant by a stable compensator. This issue called "strong stabilization" has been originally treated in [5]. The problem of the simultaneous stabilization of three or more LTI plants was initially tackled by [6] and [7]. Otherwise [8] has shown that there exists no tractable and complete conditions to check the simultaneous stabilizability of three or more systems. About the simultaneous stabilization of a segment of LTI systems, i.e. a continuum of LTI systems described by a parameter and its two endpoints, [9] has provided existence conditions which depend on the choice of the initial compensator stabilizing the extremities of the segment. For this class of system, transcendental conditions have been given by [10] and [11]. In [12] and [13] the authors address the question of the simultaneous strong stabilizability of a segment of systems. They are conditions that have been stated to stabilize each element of this family with a stable compensator. That does not imply existence conditions of a single controller stabilizing the whole set of systems belonging to this segment. Conditions for the simultaneous stabilizability of a segment of systems have been given in [14].

Concerning the robust control, Kharitonov's theorem and its generalization, [15] and [16] have been at the origin of many results. Others analysis methods have been studied in the literature but those dedicated to the synthesis of robust control with polynomial methods are only a few. In [17], the zero-exclusion principle has given conditions that are used to test the stability and allow to design robust controllers. More recently, [18], [19], [20] and [21] propose fixed-order controllers for systems with polytopic uncertainty. The methods described in these papers relied on a criterion of stability which necessitates exactly proper and strict positive realness (EP-SPR) functions. These rational functions are particular units in $H_{\infty}$ which also verify the properties of the SPR functions, see [9], [22] and [23]. Nevertheless, derived from [24], it may be easily deduced that to stabilize a polytope of systems, it is only required to synthesize units in $H_{\infty}$ whose Nyquist plots do not cut the negative real axis. Then the conditions of the EP-SPR functions are not necessary. Consequently, the constraint to make EP-SPR functions introduces sufficiency and conservatism in regard to the necessary and sufficient condition to stabilize a polytope of systems. In this paper, we propose a new approach to synthesize units in $H_{\infty}$ for stabilizing the polytopes of systems which are not SPR functions and which remain units in $H_{\infty}$ if one interchanges even (or odd) part of the numerator and denominator polynomials. The Nyquist plots of these units in $H_{\infty}$ do not cut the negative real axis, see [24].

In this manuscript, we focus on the stabilization of polytope of single input single output LTI systems. Based on the Hermite-Biehler theorem and the Edge theorem, we prove that to stabilize a polytope of systems it is sufficient to stabilize all its vertices with a simultaneous controller giving characteristic polynomials that have a common even (or odd) part. Moreover, we show that the synthesized units in $H_{\infty}$ do not cut the real negative axis of the Nyquist plot. In addition a controller design for stabilizing the polytopes of systems is provided mixing polynomial techniques and linear optimization.

The paper is organized as it follows. After preliminaries in section 2, the problem of the robust stabilization of a polytope of systems is stated in section 3 by applying the Edge theorem. Then an approach to stabilize the vertices of a polytope of systems with a simultaneous compensator is given in section 4 that leads to a simple linear programming (LP) problem. The issue of the stabilization of a polytope of systems by a simultaneous controller is formulated as positivity conditions if particular pole placement constraints hold. Finally in section 5, examples are given to illustrate this framework. In the appendix, our approach is extended to the case where characteristic polynomials in closed-loop have a common odd part. 


\section{Preliminaries}

Notations: The degree of a real polynomial $X$ is denoted $\delta(X) . \mathbb{P}_{m}$ denotes the set of real polynomials of degree $m$. The set of Hurwitz-stable polynomials is denoted $\mathcal{H}$. $\mathbb{R}$ is the real field. $\mathbb{C}$ is the complex field. $\mathbb{R}_{++}^{n}=\left\{x \in \mathbb{R}^{n}: x_{i}>0, \forall i\right\}$ and $\mathbb{R}_{-\{0\}}=\{x \in \mathbb{R}: x<0$ or $x>0\}$. The notation $x>0$ with $x \in \mathbb{R}^{n}$ means that each component of the vector $x$ is positive. If and only if is defined as iff. The exactly proper and strict positive realness functions are denoted as EP-SPR functions. $H_{\infty}$ is the set of proper stable rational functions. \|\| denotes the operator norm. $\mathbb{U}$ is the set of units in $H_{\infty}$ i.e. $U \in \mathbb{U}$ iff $U \in H_{\infty}$ and $U^{-1} \in H_{\infty}$.

In the sequel of this section, concepts on Hurwitz-stability of a polytope of real polynomials are presented. These results are used in section 3 to study the stability of a polytope of systems. The notion of Hurwitz-stability highlighted by [25] is approached hereafter under the angle of interlacing of real zeros.

\subsection{The Hermite-Biehler's stability.}

Let $f \in \mathbb{P}_{m}$ expanded as

$$
f(s)=f^{e}\left(s^{2}\right)+s f^{o}\left(s^{2}\right)=\sum_{i=0}^{m} \sigma_{i} s^{i}
$$

where $\sigma_{i}$ are real coefficients and $f^{e}$ and $f^{o}$ denote the even and odd parts of $f$ respectively. The polynomials $f^{e}$ and $f^{o}$ are given by

- if $\delta(f)=m=2 \ell+1$

$$
\begin{aligned}
& f^{e}(u)=\sigma_{0}+\sigma_{2} u+\ldots+\sigma_{(m-1)} u^{\ell} \\
& f^{o}(u)=\sigma_{1}+\sigma_{3} u+\ldots+\sigma_{m} u^{\ell} .
\end{aligned}
$$

- if $\delta(f)=m=2 \ell$

$$
\begin{aligned}
& f^{e}(u)=\sigma_{0}+\sigma_{2} u+\ldots+\sigma_{m} u^{\ell}, \\
& f^{o}(u)=\sigma_{1}+\sigma_{3} u+\ldots+\sigma_{(m-1)} u^{(\ell-1)} .
\end{aligned}
$$

Let us recall the Zeros Interlacing Property.

Definition 1 ([25], The Zeros Interlacing Property) Let $f(s)=f^{e}\left(s^{2}\right)+s f^{o}\left(s^{2}\right)$ be a real polynomial with $\delta(f)=m$. Assume that the roots of $f^{e}$ and $f^{o}$ are defined by the following sets

- if $\delta(f)=m=2 \ell+1$ and $\delta\left(f^{e}\right)=\ell, \delta\left(f^{o}\right)=\ell$

$$
\begin{aligned}
& \operatorname{roots}\left(f^{e}\right)=\left\{a_{1}, \ldots, a_{\ell}\right\}, \\
& \operatorname{roots}\left(f^{o}\right)=\left\{b_{1}, \ldots, b_{\ell}\right\},
\end{aligned}
$$

- if $\delta(f)=m=2 \ell$ and $\delta\left(f^{e}\right)=\ell, \delta\left(f^{o}\right)=\ell-1$

$$
\begin{aligned}
& \operatorname{roots}\left(f^{e}\right)=\left\{a_{1}, \ldots, a_{\ell}\right\}, \\
& \operatorname{roots}\left(f^{o}\right)=\left\{b_{1}, \ldots, b_{\ell-1}\right\} .
\end{aligned}
$$


Then $f^{e}$ and $f^{o}$ interlace iff

- the roots of $f^{e}$ and $f^{o}$ are real, negative, distinct and simple,

- the leading coefficients of $f^{e}$ and $f^{o}$ have the same sign,

- the $\ell$ roots of $f^{e}$ alternate with the $\ell$ or $\ell-1$ roots of $f^{o}$ as it follows.

$$
\begin{aligned}
& \text { If } \delta(f)=m=2 \ell+1 \text { then } \\
& \qquad b_{1}<a_{1}<b_{2} \ldots<b_{\ell}<a_{\ell}<0 .
\end{aligned}
$$$$
\text { If } \delta(f)=m=2 \ell \text { then }
$$

$$
a_{1}<b_{1}<\ldots<b_{\ell-1}<a_{\ell}<0
$$

Let us note that the cases $m=2 \ell+1$ or $m=2 \ell$ are considered indistinctly in this paper but to simplify the writing, the theoretical results are presented for only $m=2 \ell+1$ and an example is given section 5.1 for the two cases $m=2 \ell$ and $m=2 \ell+1$. It should be noticed that the case $m=2 \ell$ may be directly deduced from the case $m=2 \ell+1$.

The relationship between the zeros interlacing property of a real polynomial and the Hurwitzstability is emphasized by the Hermite-Biehler's theorem that we remind.

Lemma 1 ([25], Hermite-Biehler's theorem) The real polynomial $f(s)=f^{e}\left(s^{2}\right)+s f^{o}\left(s^{2}\right)$ is Hurwitz-stable (i.e. all the real parts of the roots of $f$ are strictly negative) iff $f^{e}$ and $f^{o}$ verify the Zeros Interlacing Property.

Let us write the partial fraction expansion of the real rational function $f^{o} / f^{e}$ with $f^{e}$ and $f^{o}$ satisfying (1). Consequently, from lemma 1, simple conditions for testing the Hurwitz-stability of $f$ are deduced.

Lemma 2 ([26], [27]) The polynomial $f(s)=f^{e}\left(s^{2}\right)+s f^{o}\left(s^{2}\right)$ with $f \in \mathbb{P}_{m}$ is Hurwitz-stable iff the two following conditions hold.

1. $f^{e}$ has real, simple and negative roots $\left(a_{1}, \ldots, a_{\ell}\right)$.

2. The real coefficients $c_{k}(k=0, \ldots, \ell)$ given by the expansion of $f^{o}$ as the following sum of products of factors of $f^{e}$

$$
f^{o}\left(s^{2}\right)=c_{0} f^{e}\left(s^{2}\right)+\sum_{k=1}^{\ell} c_{k} \frac{f^{e}\left(s^{2}\right)}{s^{2}-a_{k}} .
$$

are strictly positive.

Note that $c_{0}=0$ in the case where $m=2 \ell$.

Mention that all the next developments are made in function of the writing (3) with $f^{e}$ given a priori. The developments in function of $f^{o}$ are presented in the appendix section. 
Let us consider $n$ real polynomials $f_{i},(i=1, \ldots, n)$ with a a same even part $f^{e}$. Then we have $f_{i}(s)=f^{e}\left(s^{2}\right)+s f_{i}^{o}\left(s^{2}\right),(i=1, \ldots, n)$.

In association with $f_{i}$, a generalization of the Zeros Interlacing Property is now given.

Definition 2 (Common even interlacing property) Consider a finite collection of polynomials $f_{i}^{o},(i=1, \ldots, n)$ and a polynomial $f^{e}$ with their leading coefficients of same sign. Assume that for each pair $\left(f_{i}^{o}, f^{e}\right),(i=1, \ldots, n)$, the roots of $f_{i}^{o}$ and $f^{e}$ are all real, negative, distinct and simple defined by the following sets

$$
\begin{aligned}
& \operatorname{roots}\left(f_{i}^{o}\right)=\left\{b_{1}^{i}, \ldots, b_{\ell}^{i}\right\}, \\
& \operatorname{roots}\left(f^{e}\right)=\left\{a_{1}, \ldots, a_{\ell}\right\} .
\end{aligned}
$$

The polynomials $f_{i}^{o}$ have a common even interlacing $f^{e}$ iff the $\ell$ roots of each $f_{i}^{o}$ alternate with the $\ell$ roots of $f^{e}$ as below

$$
b_{1}^{i}<a_{1}<b_{2}^{i}<\ldots<b_{\ell}^{i}<a_{\ell}<0 .
$$

Remark that if the polynomials $f_{i}^{o},(i=1, \ldots, n)$ have a common interlacing $f^{e}$ this implies that for any $j$ th root, we have

$$
\max _{i \in\{1, \ldots, n\}} b_{j}^{i}<a_{j}<\min _{i \in\{1, \ldots, n\}} b_{j+1}^{i}
$$

By using the common even interlacing property given above, an extension of lemma 2 is deduced for testing the Hurwitz-stability of polynomials $f_{i},(i=1, \ldots, n)$.

Corollary 1 The polynomials $f_{i}(s)=f^{e}\left(s^{2}\right)+s f_{i}^{o}\left(s^{2}\right),(i=1, \ldots, n)$ with $f_{i} \in \mathbb{P}_{m}$ are Hurwitzstable iff the two following conditions hold.

1. $f^{e}$ has real, simple and negative roots $\left(a_{1}, \ldots, a_{\ell}\right)$.

2. The real coefficients $c_{i, k}(i=1, \ldots, n)$ and $(k=0, \ldots, \ell)$ given by the expansions of $f_{i}^{o}$ $(i=1, \ldots, n)$ as the following sums of products of factors of $f^{e}$

$$
f_{i}^{o}\left(s^{2}\right)=c_{i, 0} f^{e}\left(s^{2}\right)+\sum_{k=1}^{\ell} c_{i, k} \frac{f^{e}\left(s^{2}\right)}{s^{2}-a_{k}} .
$$

are strictly positive.

Proof: That is a consequence of lemma 2. 


\subsection{Matrix formulation of $f_{i}^{o}$ and $f^{e}$.}

Let us set $f^{e}\left(s^{2}\right)=\gamma^{e} \prod_{j=1}^{\ell}\left(s^{2}-a_{j}\right)$ with $\gamma^{e} \in \mathbb{R}_{-\{0\}}$. Consequently relation (4) can be simplified as it follows

$$
\begin{aligned}
f_{i}^{o}\left(s^{2}\right) & =\gamma^{e} c_{i, 0} \prod_{j=1}^{\ell}\left(s^{2}-a_{j}\right)+\gamma^{e} \sum_{k=1}^{\ell} c_{i, k} \prod_{\substack{j=1 \\
j \neq k}}^{\ell}\left(s^{2}-a_{j}\right) \\
& =\sum_{k=0}^{\ell} c_{i, k} \sum_{j=0}^{\ell-1} v_{j, k} s^{2 j} \\
& =V A^{e} \Psi_{i}^{e}, \quad i=1, \ldots, n . \\
f^{e}\left(s^{2}\right) & =V H^{e}
\end{aligned}
$$

where

$$
\begin{aligned}
\Psi_{i}^{e} & =\left[\begin{array}{lll}
c_{i, 0} & \ldots & c_{i, \ell}
\end{array}\right]^{T} \\
V & =\left[\begin{array}{llll}
1 & s^{2} & \ldots & s^{2 \ell}
\end{array}\right] \\
A^{e} & =\left[\begin{array}{cccc}
v_{0,0} & v_{0,1} & \ldots & v_{0, \ell} \\
v_{1,0} & v_{1,1} & \ldots & v_{1, \ell} \\
\vdots & \vdots & \vdots & \vdots \\
v_{\ell, 0} & v_{\ell, 1} & \ldots & v_{\ell, \ell}
\end{array}\right] \\
H^{e} & =\left[\begin{array}{llll}
h_{0}^{e} & h_{1}^{e} & \ldots & h_{\ell}^{e}
\end{array}\right]^{T}
\end{aligned}
$$

Note that matrix $A^{e}$ only depends on the even part $f^{e}$.

By using the above notations, a reformulation of corollary 1 is now deduced.

Corollary 2 The polynomials $f_{i} \in \mathbb{P}_{m},(i=1, \ldots, n)$ are Hurwitz-stable iff the two following conditions hold

1. $f^{e}$ is a polynomial with real, simple and negative roots $\left(a_{1}, \ldots, a_{\ell}\right)$.

2. There exist $\Psi_{i}^{e} \in \mathbb{R}_{++}^{\ell+1}$ for $i=1, \ldots, n$, given by (6a).

Proof: That is a consequence of the writing (4) as (5a).

\subsection{Hurwitz-stability of a polytope of real polynomials.}

Let $S_{f}$ be a convex polytope of real polynomials described by the set of $n$ vertices $f_{i} \in \mathbb{P}_{m}$ as it follows

$$
S_{f} \triangleq\left\{f: f=\sum_{i=1}^{n} \lambda_{i} f_{i}, \lambda_{i} \geq 0, \sum_{i=1}^{n} \lambda_{i}=1\right\}
$$


The set of the exposed edges ${ }^{1}$ connecting two vertices $f_{i}$ and $f_{j}$ in $\mathbb{P}_{m}$ of the polytope of polynomials $S_{f}$ is defined by $S_{f_{i, j}}$ where

$$
S_{f_{i, j}} \triangleq\left\{f_{i, j}: f_{i, j}=\alpha f_{i}+(1-\alpha) f_{j}, \alpha \in[0,1], i=1, \ldots, n-1, j=i+1, \ldots, n\right\}
$$

Recall theorem 1 in [2] that shows that the stability of a polytope of real polynomials can be inferred from its exposed edges.

Theorem 1 ([2]) The polytope of real polynomials $S_{f}$ is Hurwitz-stable iff the set of exposed edges $S_{f_{i, j}}$ are Hurwitz-stable.

The Hurwitz-stability issue of $S_{f}$ may be simplified by considering corollary 3 in [28].

Lemma 3 ([28]) Let $f_{i} \in \mathbb{P}_{m}$ and $f_{j} \in \mathbb{P}_{m}$ be two vertices of $S_{f}$ with their leading coefficients of same sign and with a same even part $f^{e}$, then the exposed edge $f_{i, j}$ defined by (8) is Hurwitz-stable iff $f_{i}$ and $f_{j}$ are Hurwitz-stable.

Corollary 3 If the vertices $f_{i} \in \mathbb{P}_{m},(i=1, \ldots, n)$ of a polytope of real polynomials $S_{f}$ are Hurwitz-stable with a same even part $f^{e}$ and if the leading coefficients of $f_{i},(i=1, \ldots, n)$ have a same sign then the polytope of real polynomials $S_{f}$ is Hurwitz-stable.

Proof: A consequence of theorem 1 and lemma 3.

In connection with corollary 2 , a reformulation of corollary 3 is now deduced.

Corollary 4 Consider a convex polytope of real polynomials $S_{f}$ defined by the set of vertices $f_{i} \in \mathbb{P}_{m}$ verifying (7) with a same even part $f^{e}$. If the two following conditions hold then $S_{f}$ is Hurwitz-stable.

1. $f^{e}$ is a polynomial with real, simple and negative roots $\left(a_{1}, \ldots, a_{\ell}\right)$.

2. There exist $\Psi_{i}^{e} \in \mathbb{R}_{++}^{\ell+1}(i=1 \ldots n)$ given by (6a).

Proof: As a consequence of corollaries 2 and 3.

Lemma 4 Let $S_{f}$ be a convex polytope of real polynomials described by a set of $n$ vertices $f_{i} \in \mathbb{P}_{m}$ verifying (7). The number of exposed edges of $S_{f}$ is $\bar{n}=\sum_{i=1}^{n-1}(n-i)$.

Proof: Consider $f_{i},(i=1, \ldots, n)$, vertices of a convex polytope $S_{f}$. Count the number of different exposed edges connected to each vertex with $f_{i, j}=f_{j, i}$. Each vertex $f_{i},(i=1, \ldots, n-1)$, is connected by $n-i$ different exposed edges to $n-i$ vertices. In consequence, the total number of exposed edges of $S_{f}$ is $\bar{n}=\sum_{i=1}^{n-1}(n-i)$.

Note that the problem of Hurwitz-stability of a polytope of real polynomials $S_{f}$ is significantly reduced by considering corollary 3 and lemma 4, with which we only need to verify the Hurwitzstability of $n$ vertices of $S_{f}$ instead to verify the Hurwitz-stability of $\bar{n}$ exposed edges of $S_{f}, n<\bar{n}$ if $n>3$.

\footnotetext{
${ }^{1}$ See [2] for the definition of the exposed edges.
} 


\section{Robust stabilization of a polytope of plants.}

Now a robust stability analysis for a polytope of systems may be carried out.

Definition 3 (A polytope of plants) The convex polytope of plants $\Omega_{G}$ generated by $n$ SISO LTI systems $G_{i}=N_{i} D_{i}^{-1}(i=1 \ldots n)$ is defined as

$$
\Omega_{G} \triangleq\left\{G: G=N D^{-1}, N=\sum_{i=1}^{n} \lambda_{i} N_{i}, D=\sum_{i=1}^{n} \lambda_{i} D_{i}, \lambda_{i} \geqslant 0, \sum_{i=1}^{n} \lambda_{i}=1\right\}
$$

where $N_{i}$ and $D_{i}$ are real polynomials with $\delta\left(N_{i}\right) \leqslant \delta\left(D_{i}\right)$ and $D_{i} \in \mathbb{P}_{d}$.

All pairs of vertices $\left(G_{i}, G_{j}\right)$, with $i=1, \ldots, n-1$ and $j=i+1, \ldots, n$, of a polytope of systems $\Omega_{G}$ are connected by an exposed edge $G_{i, j}$. Each exposed edge is a segment of systems. The set of all segments of systems of $\Omega_{G}$ is defined as the set $\Omega_{G_{i, j}}$ given below.

Definition 4 (The set of segments of systems $\Omega_{G_{i, j}}$ ) The set of segments of systems $\Omega_{G_{i, j}}$ connecting any two vertices of the polytope of systems $\Omega_{G}$ is defined as

$$
\begin{gathered}
\Omega_{G_{i, j}} \triangleq\left\{G_{i, j}: G_{i, j}=N_{i, j} D_{i, j}^{-1}, N_{i, j}=\alpha N_{i}+(1-\alpha) N_{j}, D_{i, j}=\alpha D_{i}+(1-\alpha) D_{j},\right. \\
\alpha \in[0,1], i=1, \ldots, n-1, j=i+1, \ldots, n\} .
\end{gathered}
$$

The objective of this paper is to propose a proper controller $C=X Y^{-1}$ that stabilizes the polytope of systems $\Omega_{G}$ where $X$ and $Y$ are real polynomials. Consequently, the existence conditions of a compensator $C$ satisfying

$$
\Phi(G, C)=(N X+D Y) \in \mathcal{H}
$$

are studied.

Lemma 5 The compensator $C$ stabilizes the polytope of systems $\Omega_{G}$ iff $C$ stabilizes the set of segments of systems $\Omega_{G_{i, j}}$.

Proof: The compensator $C$ stabilizes the polytope of systems $\Omega_{G}$ iff

$$
\Phi(G, C)=N X+Y D=\left(\sum_{i=1}^{n} \lambda_{i}\left(N_{i} X+D_{i} Y\right)\right) \in \mathcal{H}
$$

or iff

$$
\left(\sum_{i=1}^{n} \lambda_{i} \Phi\left(G_{i}, C\right)\right) \in \mathcal{H} \text { with } \lambda_{i} \geq 0, \sum_{i=1}^{n} \lambda_{i}=1
$$

Hence, the compensator $C$ stabilizes the polytope of systems $\Omega_{G}$ iff the polytope of polynomials $\phi$ is Hurwitz-stable where

$$
\phi \triangleq\left\{\Phi(G, C): \Phi(G, C)=\sum_{i=1}^{n} \lambda_{i} \Phi\left(G_{i}, C\right), \lambda_{i} \geq 0, \sum_{i=1}^{n} \lambda_{i}=1\right\}
$$


By applying theorem 1, we know that $\Phi(G, C) \in \mathcal{H}$ iff $\Phi\left(G_{i, j}, C\right) \in \mathcal{H}$. This implies that $\phi$ is Hurwitz-stable iff $\phi_{i, j}$ is Hurwitz-stable with $\phi_{i, j}$ defined as the set of exposed edges of $\phi$ where

$$
\begin{gathered}
\phi_{i, j} \triangleq\left\{\Phi\left(G_{i, j}, C\right): \Phi\left(G_{i, j}, C\right)=\alpha \Phi\left(G_{i}, C\right)+(1-\alpha) \Phi\left(G_{j}, C\right),\right. \\
\alpha \in[0,1], i=1, \ldots, n-1, j=i+1, \ldots, n\} .
\end{gathered}
$$

Consequently, the controller $C$ stabilizes the polytope of plants $\Omega_{G}$ iff $C$ stabilizes the set of segments of systems $\Omega_{G_{i, j}}$ defined by (10).

Now to conclude this section, we can observe that by applying corollary 3 to the polytope of polynomials $\phi$, we can simplify the issue of the Hurwitzness of $\phi$. That is, if the vertices $\Phi\left(G_{i}, C\right)$ $(i=1, \ldots n)$ of the polytope $\phi$ are Hurwitz-stable with a same degree and with their leading coefficients of same sign and a same even part $\Phi\left(G_{i}, C\right)^{e}$ where

$$
\Phi\left(G_{i}, C\right)(s)=\Phi\left(G_{i}, C\right)^{e}\left(s^{2}\right)+s \Phi\left(G_{i}, C\right)^{o}\left(s^{2}\right)
$$

then the polytope of polynomials $\phi$ is Hurwitz-stable.

\section{Computational conditions for robust stabilization of a polytope of plants with a simultaneous compensator.}

In this section, the problem of the stabilization of the polytope of systems $\Omega_{G}$ is posed as that of the stabilization of its vertices $G_{i}$ with a simultaneous compensator $C$ that guarantees a same even part $\Phi\left(G_{i}, C\right)^{e}$ for the closed-loop characteristic polynomials associated with $G_{i}$ for $i=1, \ldots, n$.

Theorem 2 Consider a convex polytope of plants $\Omega_{G}$ defined by (9). If there exists a simultaneous compensator $C$ satisfying the following relations

$$
\begin{aligned}
& \Phi\left(G_{i}, C\right) \in \mathcal{H}, \\
& \Phi\left(G_{i}, C\right)^{e}=\Phi\left(G_{j}, C\right)^{e}, i=1, \ldots, n-1, j=i+1, \ldots, n .
\end{aligned}
$$

with the leading coefficients of $\Phi\left(G_{i}, C\right)$ of same sign, then $C$ stabilizes $\Omega_{G}$.

Proof: This theorem is a consequence of lemma 5 and corollary 3.

Henceforth, the issue of the stabilization of the polytope of systems $\Omega_{G}$ is reduced to the design of a simultaneous compensator that must satisfy the particular poles placements required by theorem 2 . This yields to the following lemma.

Lemma 6 Consider a convex polytope of plants $\Omega_{G}$ defined by (9). Let $V, A^{e}, H^{e}$ and $\Psi_{i}^{e}$ be given by (6b), (6c), (6d) and (6a) respectively. If there exist $\Psi_{i}^{e} \in \mathbb{R}_{++}^{\ell+1}(i=1, \ldots, n)$ and a compensator $C$ verifying the following relations

$$
\begin{aligned}
& \Phi\left(G_{i}, C\right)^{e}=V H^{e}, \quad i=1, \ldots, n \\
& \Phi\left(G_{i}, C\right)^{o}=V A^{e} \Psi_{i}^{e}
\end{aligned}
$$

then $C$ stabilizes $\Omega_{G}$. 
Proof: This lemma is a consequence of corollary 4 and theorem 2.

Notice that the simultaneous controller $C$ proposed in lemma 6 has a fixed order since the degree of the closed-loop characteristic polynomials $\Phi\left(G_{i}, C\right)$ associated with the vertices of the polytope of polynomials $\phi$ given in (12), are chosen a priori.

Consider the simultaneous compensator $C=X Y^{-1}$ and the vertices $G_{i}=N_{i} D_{i}^{-1}(i=1, \ldots, n)$ of a polytope of systems $\Omega_{G}$ where $X, Y, N_{i}$ and $D_{i}$ be real polynomials expressed in terms of their even and odd parts as follows

$$
\begin{array}{r}
X(s)=X^{e}\left(s^{2}\right)+s X^{o}\left(s^{2}\right), \quad Y(s)=Y^{e}\left(s^{2}\right)+s Y^{o}\left(s^{2}\right) \\
N_{i}(s)=N_{i}^{e}\left(s^{2}\right)+s N_{i}^{o}\left(s^{2}\right), \quad D_{i}(s)=D_{i}^{e}\left(s^{2}\right)+s D_{i}^{o}\left(s^{2}\right)
\end{array}
$$

Then we obtain

$$
\begin{aligned}
\Phi\left(G_{i}, C\right)^{e} & =\Gamma_{i}^{e} C_{p}, \quad i=1, \ldots, n \\
\Phi\left(G_{i}, C\right)^{o} & =\Gamma_{i}^{o} C_{p}
\end{aligned}
$$

with

$$
\begin{aligned}
C_{p}\left(s^{2}\right) & =\left[\begin{array}{llll}
X^{e}\left(s^{2}\right) & Y^{e}\left(s^{2}\right) & X^{o}\left(s^{2}\right) & Y^{o}\left(s^{2}\right)
\end{array}\right]^{T} \\
\Gamma_{i}^{e}\left(s^{2}\right) & =\left[\begin{array}{llll}
N_{i}^{e}\left(s^{2}\right) & D_{i}^{e}\left(s^{2}\right) & s^{2} N_{i}^{o}\left(s^{2}\right) & s^{2} D_{i}^{o}\left(s^{2}\right)
\end{array}\right] \\
\Gamma_{i}^{o}\left(s^{2}\right) & =\left[\begin{array}{llll}
N_{i}^{o}\left(s^{2}\right) & D_{i}^{o}\left(s^{2}\right) & N_{i}^{e}\left(s^{2}\right) & D_{i}^{e}\left(s^{2}\right)
\end{array}\right]
\end{aligned}
$$

Reformulate $\Phi\left(G_{i}, C\right)^{e}$ and $\Phi\left(G_{i}, C\right)^{o}$ in a matrix form. For that consider

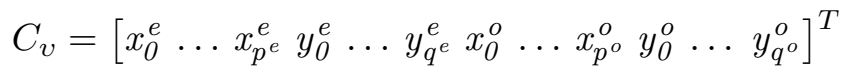

with $p^{e}=\delta\left(X^{e}\right), p^{o}=\delta\left(X^{o}\right), q^{e}=\delta\left(Y^{e}\right)$ and $q^{o}=\delta\left(Y^{o}\right)$.

Define two matrices $\Lambda_{i}^{e}$ and $\Lambda_{i}^{o}$ such that

$$
\begin{aligned}
& \Phi\left(G_{i}, C\right)^{e}=V \Lambda_{i}^{e} C_{v}, \quad i=1, \ldots, n \\
& \Phi\left(G_{i}, C\right)^{o}=V \Lambda_{i}^{o} C_{v}
\end{aligned}
$$

where $\Lambda_{i}^{e}$ and $\Lambda_{i}^{o}$ are a rewriting of $\Gamma_{i}^{e}$ and $\Gamma_{i}^{o}$ according to the coefficients of polynomials $N_{i}^{e}, D_{i}^{e}$, $N_{i}^{o}$ and $D_{i}^{o}$.

Lemma 6 and relations (18) permit to deduce the following lemma.

Lemma 7 Consider a convex polytope of plants $\Omega_{G}$ defined by (9). Let $A^{e}, H^{e}$ and $\Psi_{i}^{e}$ given by (6c), (6d) and (6a) respectively. If there exist $C_{v}$ and $\Psi_{i}^{e} \in \mathbb{R}_{++}^{\ell+1},(i=1, \ldots, n)$ satisfying the following relations

$$
\begin{aligned}
\Lambda_{i}^{e} C_{v} & =H^{e} \quad i=1, \ldots, n, \\
\Lambda_{i}^{o} C_{v} & =A^{e} \Psi_{i}^{e}
\end{aligned}
$$

then $C$ stabilizes $\Omega_{G}$. 
Proof: This result is a consequence of lemma 6 and relations (18a) and (18b).

Now rewrite relations (19)

$$
B^{e}=\Theta^{e} \chi^{e}
$$

where

$$
\begin{aligned}
& B^{e}=\left[\begin{array}{c}
0 \\
\vdots \\
0 \\
H^{e} \\
H^{e} \\
\vdots \\
H^{e}
\end{array}\right], \Theta^{e}=\left[\begin{array}{ccccc}
A^{e} & 0 & \ldots & 0 & -\Lambda_{1}^{o} \\
\vdots & \vdots & \ddots & \vdots & \vdots \\
0 & 0 & \ddots & A^{e} & -\Lambda_{n}^{o} \\
0 & 0 & \ldots & 0 & \Lambda_{1}^{e} \\
0 & 0 & \ldots & 0 & \Lambda_{2}^{e} \\
\vdots & \vdots & \ldots & \vdots & \vdots \\
0 & 0 & \ldots & 0 & \Lambda_{n}^{e}
\end{array}\right] \\
& \chi^{e}=\left[\begin{array}{ll}
\psi^{e T} & C_{v}^{T}
\end{array}\right]^{T}, \\
& \psi^{e}=\left[\begin{array}{lllll}
\Psi_{1}^{e T} & \Psi_{2}^{e T} & \Psi_{3}^{e T} & \ldots & \Psi_{n}^{e T}
\end{array}\right]^{T} \in \mathbb{R}_{++}^{n(\ell+1)} .
\end{aligned}
$$

There is solutions $\psi^{e} \in \mathbb{R}^{n(\ell+1)}$ and $C_{v}$ to the equation (20) iff [29].

$$
\operatorname{rank}\left(\left[\begin{array}{cc}
B^{e} & \Theta^{e}
\end{array}\right]\right)=\operatorname{rank}\left(\Theta^{e}\right) .
$$

By considering equation (19), the robust stabilization of the polytope of systems $\Omega_{G}$ given by lemma 7 is now set as the issue of feasibility of a linear programming problem (LP).

Theorem 3 Let $\Omega_{G}$ be a convex polytope of plants defined by (9). Assume that (21) holds then the controller $C$ stabilizes $\Omega_{G}$ if there exists a solution $\chi^{e}$ to the linear programming (LP) problem given by (20).

Proof: Since (20) is a rewritten of the relations (19), then theorem 3 is equivalent to lemma 7.

This LP problem can be expressed as a LP computational procedure. Readily available, linear programming software allows to solve this type of system (20) in an efficient way. This possibility is demonstrated through some numerical examples that are reported in the section 5 .

After focusing initially on the controller design for stabilizing a polytope of systems, we are again going to review some literature results. Derived from [24], an equivalent condition to the one given in lemma 5 may be formulated.

Corollary 5 To stabilize a polytope of plants $\Omega_{G}$ with a LTI compensator, it is necessary and sufficient that the units in $H_{\infty}$ formed by the ratio of every two closed-loop characteristic polynomials associated with the vertices of the polytope of systems have the modulus of their argument less than $180^{\circ}$. 
Proof: This necessary and sufficient condition is a direct consequence deduced from lemma 5 and theorem 2.3 in [24].

The condition given in corollary 5 may be rewritten as

$$
\left|\arg \left(\frac{\Phi\left(G_{i}, C\right)(j w)}{\Phi\left(G_{j}, C\right)(j w)}\right)\right|<180^{\circ}, \quad i=1, \ldots, n-1, j=i+1, \ldots, n
$$

with

$$
\frac{\Phi\left(G_{i}, C\right)}{\Phi\left(G_{j}, C\right)} \in \mathbb{U}
$$

The approach outlined here, permits to synthesize units in $H_{\infty}$ which remain units in $H_{\infty}$ if one interchanges even (or odd) part of the numerator and denominator polynomials. The rational

functions $\frac{\Phi\left(G_{i}, C\right)}{\Phi\left(G_{j}, C\right)}$ may have the modulus of their argument more than $90^{\circ}$, see [24]. Consequently the framework described in this article for the stabilization of polytopes of systems allows to envisage other solutions than those allowed by [18].

\section{$5 \quad$ Illustrative examples}

Several examples are now provided to illustrate the applicability of our approach. Since the interval plants are an important class of systems considered in many applications, we begin in the sections 5.1 and 5.2 to apply our approach on these particular uncertain systems. In the last part, section 5.3 , an application is given for a polytope of systems that does not belong to the class of interval systems. That is a system whose the polynomial coefficients are not bound by some minimum and maximum values. On the other hand these coefficients can be dependant.

Although our analysis and our conditions of robust stability given in the sections 3 and 4 do not fall within the framework of the so-called "16-plant theorem", see [30], [31], we show by these examples that we can use our approach to stabilize these polytopes of systems.

\subsection{A detailed example}

To illustrate this design as defined in section 4 , we propose to stabilize an interval plant $\widetilde{G}$ with a proper LTI compensator where

$$
\widetilde{G}(s)=\frac{s^{2}+n_{0}}{2 s^{2}+d_{1} s+1}
$$

with $n_{0} \in\left[\epsilon_{\min }, \epsilon_{\max }\right]$ and $d_{1} \in\left[\eta_{\min }, \eta_{\max }\right]$.

First of all, if we consider a simple gain $K$ as compensator, the characteristic equation of the closed-loop system is

$$
\Phi(\widetilde{G}, K)(s)=(2+K) s^{2}+d_{1} s+\left(1+K n_{0}\right)
$$


We observe that a constant gain $K$ cannot stabilize a such system $\widetilde{G}$ if $d_{1}$ has not always the same sign.

Before developing our approach to synthesize a robust controller stabilizing $\widetilde{G}$, the following result is stated.

Proposition 1: Consider the interval plant $\widetilde{G}$ given by $(24)$. For all $n_{0} \in\left[\epsilon_{\min }, \epsilon_{\max }\right]$ and $d_{1} \in\left[\eta_{\min }, \eta_{\max }\right]$, there exists a polytope of plants $\Omega_{G}$ defined by its vertices $G_{i}=N_{i} D_{i}^{-1}(i=1 \ldots 4)$ where

$$
\begin{aligned}
& G_{1}(s)=\frac{s^{2}+\epsilon_{\min }}{2 s^{2}+\eta_{\min } s+1}, \quad G_{2}(s)=\frac{s^{2}+\epsilon_{\min }}{2 s^{2}+\eta_{\max } s+1} \\
& G_{3}(s)=\frac{s^{2}+\epsilon_{\max }}{2 s^{2}+\eta_{\min } s+1}, \quad G_{4}(s)=\frac{s^{2}+\epsilon_{\max }}{2 s^{2}+\eta_{\max } s+1}
\end{aligned}
$$

such that $\widetilde{G} \in \Omega_{G}$.

Proof: Let $G=N D^{-1}$ with

$$
N=\sum_{i=1}^{4} \lambda_{i} N_{i}, D=\sum_{i=1}^{4} \lambda_{i} D_{i}, \sum_{i=1}^{4} \lambda_{i}=1 \text { and } \lambda_{i} \geq 0
$$

Or equivalently, we have

$$
\begin{aligned}
& N(s)=s^{2}+\left(\lambda_{1}+\lambda_{2}\right) \epsilon_{\min }+\left(\lambda_{3}+\lambda_{4}\right) \epsilon_{\max } \\
& D(s)=2 s^{2}+\left(\lambda_{1}+\lambda_{3}\right) \eta_{\min } s+\left(\lambda_{2}+\lambda_{4}\right) \eta_{\max } s+1 \\
& \text { with } \sum_{1}^{4} \lambda_{i}=1 \quad \text { and } \quad \lambda_{i} \geq 0, i=1 \ldots 4
\end{aligned}
$$

Using the above relations, we can define the scalars $\alpha_{j} \in[0,1](j=1,2)$ that satisfy the following relations

$$
\left\{\begin{aligned}
\alpha_{1} & =\lambda_{1}+\lambda_{2} \\
1-\alpha_{1} & =\lambda_{3}+\lambda_{4} \\
\alpha_{2} & =\lambda_{1}+\lambda_{3} \\
1-\alpha_{2} & =\lambda_{2}+\lambda_{4}
\end{aligned}\right.
$$

Now let us show that for any $\alpha_{j} \in[0,1](j=1,2)$, there exist $\lambda_{i}(i=1 \ldots, 4)$ with $0 \leq \lambda_{i}$ and $\sum_{i=1}^{4} \lambda_{i}=1$. In terms of $(26)$, let us remark that for any $\alpha_{j} \in[0,1](j=1,2)$, we can always choose $0 \leq \lambda_{1} \leq 1$ such that the following inequalities hold. 


$$
\begin{cases}0 \leq \lambda_{1} & \leq \alpha_{1} \leq 1 \\ 0 \leq \lambda_{1} & \leq \alpha_{2} \leq 1 \\ -1 \leq \alpha_{1}+\alpha_{2}-1 & \leq \lambda_{1} \leq 1\end{cases}
$$

From relations (26) and (27), we deduce that for any $\alpha_{j} \in[0,1](j=1,2)$, there exist $\lambda_{2} \geq 0$, $\lambda_{3} \geq 0$ and $\lambda_{4} \geq 0$ with $\sum_{1}^{4} \lambda_{i}=1$ that the following relationships hold.

$$
\begin{cases}0 \leq \lambda_{2}=\alpha_{1}-\lambda_{1} & \leq 1 \\ 0 \leq \lambda_{3}=\alpha_{2}-\lambda_{1} & \leq 1 \\ 0 \leq \lambda_{4}=1-\alpha_{1}-\alpha_{2}+\lambda_{1} & \leq 1\end{cases}
$$

Then we can rewrite $\widetilde{G}=\widetilde{N} \widetilde{D}^{-1}$ with

$$
\begin{gathered}
\widetilde{N}(s)=s^{2}+\left(\alpha_{1} \epsilon_{\min }+\left(1-\alpha_{1}\right) \epsilon_{\max }\right) \\
\widetilde{D}(s)=2 s^{2}+\left(\alpha_{2} \eta_{\min }+\left(1-\alpha_{2}\right) \eta_{\max }\right) s+1 \\
\alpha_{1} \in[0,1], \quad \alpha_{2} \in[0,1]
\end{gathered}
$$

Notice that for any $\alpha_{j} \in[0,1],(j=1,2)$, there exist always scalars $\lambda_{i},(i=1,4)$ such that $0 \leq \lambda_{i} \leq 1,(i=1, \ldots, 4)$ that satisfy $(27)$ and (28) by taking $\lambda_{1}=\min \left(\alpha_{1}, \alpha_{2}\right)$.

Now consider $\widetilde{G}$ defined by $(24)$ with $\epsilon_{\min }=1, \epsilon_{\max }=2, \eta_{\min }=-1, \eta_{\max }=1$. By applying Proposition 1, we know that if a compensator $C$ exists that stabilizes the polytope of plants $\Omega_{G}$ defined by its four vertices $(25)$ then this compensator stabilizes $\widetilde{G}$. We can note that for stabilizing the polytope of systems $\Omega_{G}$, it is sufficient to stabilize simultaneously only the four vertices (25) instead of six segments of systems as in the case of lemma 5. Consequently, to synthesize a robust controller that stabilizes $\Omega_{G}$, theorem 3 and theorem 5 will be considered successively.

Firstly, let us solve this robust stabilization problem guaranteeing that the closed-loop characteristic polynomials associated with the four vertices of $\Omega_{G}$ have the same even part. To that goal, we can take a controller of the following form

$$
C^{e}(s)=\frac{x_{1} s}{y_{2} s^{2}+y_{0}}
$$

We choose two negative distinct real roots: $a_{1}=-0.5, a_{2}=-4$. Then we find

$$
\psi=\left[\begin{array}{llllllll}
3.06 & 0.01 & 2.04 & 1.03 & 3.06 & 1.01 & 2.04 & 2.03
\end{array}\right]
$$

and we get

$$
C^{e}(s)=\frac{3.57 s}{0.5 s^{2}+2}
$$

The closed-loop characteristic polynomials associated with the four vertices of $\Omega_{G}$ are Hurwitzstable where 


$$
\begin{aligned}
& \Phi\left(G_{1}, C^{e}\right)(s)=s^{4}+3.07 s^{3}+4.5 s^{2}+1.57 s+2 \\
& \Phi\left(G_{2}, C^{e}\right)(s)=s^{4}+3.07 s^{3}+4.5 s^{2}+5.14 s+2 \\
& \Phi\left(G_{3}, C^{e}\right)(s)=s^{4}+4.07 s^{3}+4.5 s^{2}+5.57 s+2 \\
& \Phi\left(G_{4}, C^{e}\right)(s)=s^{4}+4.07 s^{3}+4.5 s^{2}+9.14 s+2
\end{aligned}
$$

Now solve the same problem that the one that we have just treated but by considering theorem 5 . With this formulation detailed in the appendix section, we can compute a controller $C^{o}$ guaranteeing that the closed-loop characteristic polynomials associated with the four vertices of $\Omega_{G}$ have the same odd part. To that goal, we can consider a controller of the form

$$
C^{o}(s)=\frac{x_{2} s^{2}+x_{0}}{y_{3} s^{3}+y_{1} s}
$$

The odd part of the characteristic polynomials can be fixed by the two negative distinct real roots $b_{1}=-2.3704, b_{2}=-0.5$. This yields to

$$
\psi=\left[\begin{array}{llllllllllll}
3.5926 & 0.198 & 6.8363 & 3.5926 & 1.0941 & 1.8476 & 4.5926 & 0.6980 & 6.8363 & 4.5926 & 1.5941 & 1.8476
\end{array}\right]
$$

and

$$
C^{o}(s)=\frac{221 s^{2}+20}{27 s^{3}+64 s}
$$

The closed-loop characteristic polynomials associated with the four vertices of the polytope $\Omega_{G}$ are Hurwitz-stable where

$$
\begin{aligned}
& \Phi\left(G_{1}, C^{o}\right)(s)=54 s^{5}+194 s^{4}+155 s^{3}+177 s^{2}+64 s+20 \\
& \Phi\left(G_{2}, C^{o}\right)(s)=54 s^{5}+194 s^{4}+155 s^{3}+398 s^{2}+64 s+40 \\
& \Phi\left(G_{3}, C^{o}\right)(s)=54 s^{5}+248 s^{4}+155 s^{3}+305 s^{2}+64 s+20 \\
& \Phi\left(G_{4}, C^{o}\right)(s)=54 s^{5}+248 s^{4}+155 s^{3}+526 s^{2}+64 s+40
\end{aligned}
$$

Consider $U_{i, j}^{o}=\frac{\Phi\left(G_{i}, C^{o}\right)}{\Phi\left(G_{j}, C^{o}\right)}$ and $U_{i, j}^{e}=\frac{\Phi\left(G_{i}, C^{e}\right)}{\Phi\left(G_{j}, C^{e}\right)}$ with $i=1, \ldots, 3, j=i+1, \ldots, 4$. Let us plot the modulus of the argument of the rational functions $U_{i, j}(j w)^{e}$ and $U_{i, j}(j w)^{o}$, see figures 1 and 2. We observe that all these units have the modulus of their argument less than $180^{\circ}$ and some of these units have modulus of their argument more than $90^{\circ}$. Hence, they are not all EP-SPR functions.

The conditions given in the theorems 2 and 4 are all satisfied then $C^{e}$ and $C^{o}$ stabilize the polytope of systems $\Omega_{G}$. Accordingly, $C^{e}$ and $C^{o}$ stabilize $\widetilde{G}$. 

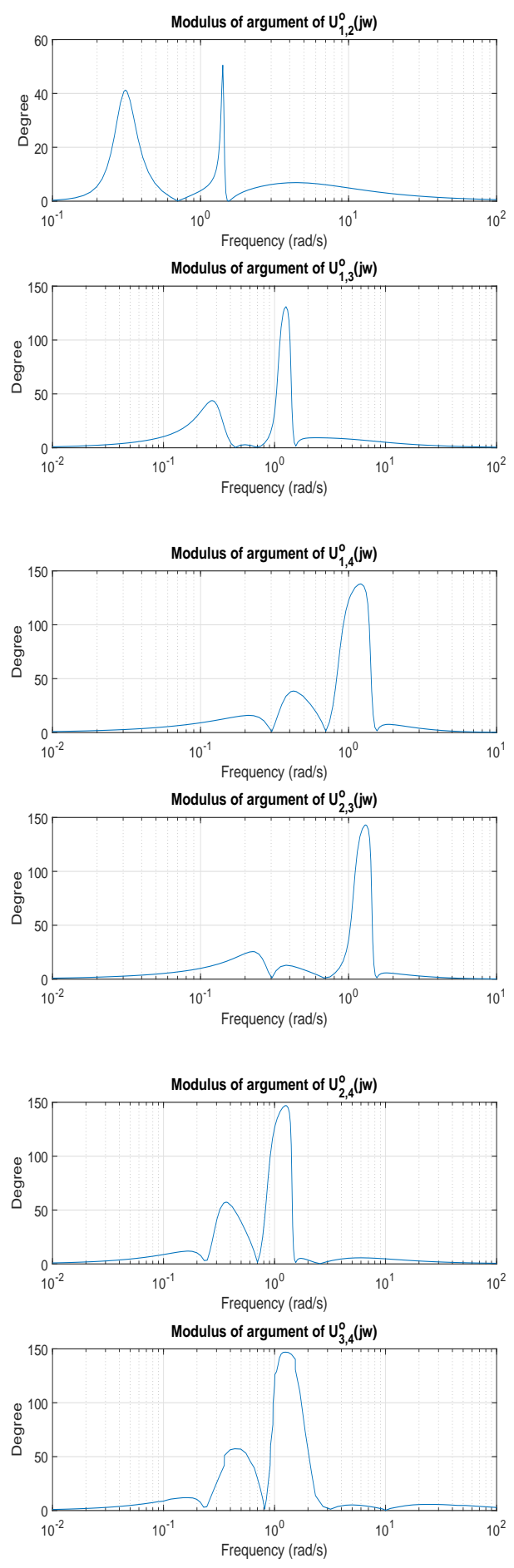

Fig 1: Plot of $\left|\arg U_{i, j}^{o}(j w)\right|$ in regard to $C_{o}$
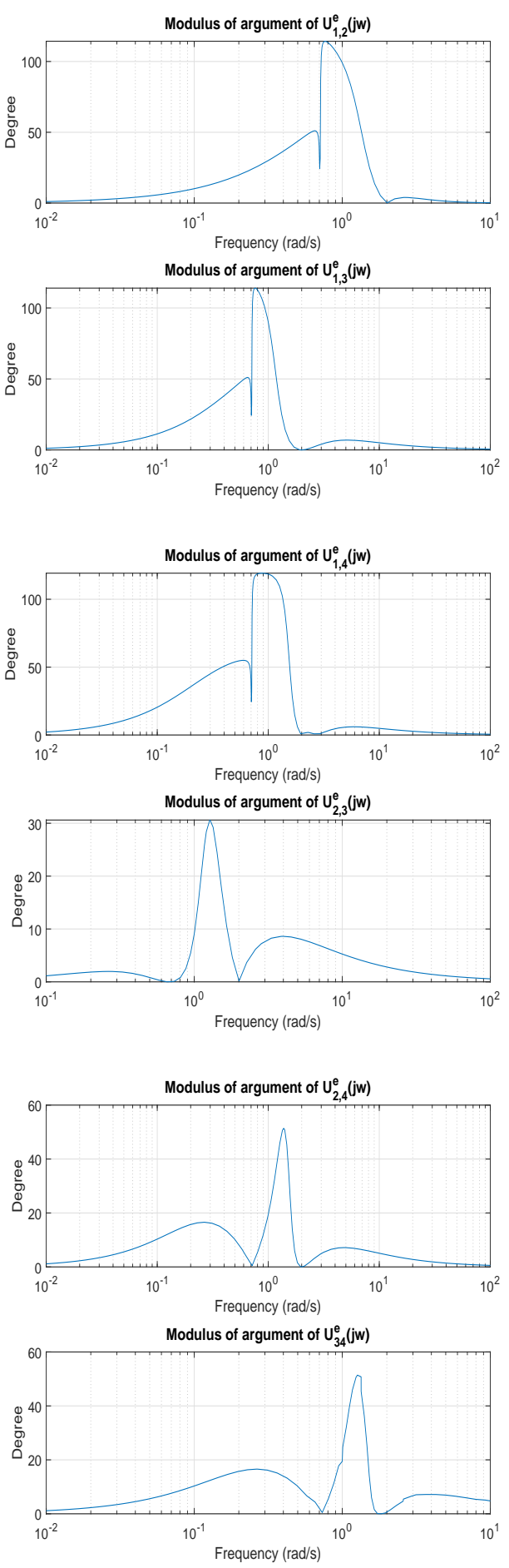

Fig 2: Plot of $\left|\arg U_{i, j}^{e}(j w)\right|$ in regard to $C_{e}$ 


\subsection{A numerical benchmark}

In this part, a collection of interval plants are now presented showing the efficiency of our numerical design when the order of these systems grows. Consequently, the number of constraints treated in these configurations by the linear programming procedure becomes important. It is about pointing out that in spite of this numerical complexity, the algorithm can find feasible solutions. The allowed maximum uncertainty is defined by a parameter $\tau_{\max }$ which is linked to the size of the uncertainty intervals with $0 \leq \tau_{\max }$. To simplify the presentation, only simultaneous compensators guaranteeing a same even part for the characteristic polynomials are given.

\subsubsection{Example 1}

A similar example to the one given previously in section 5.1 but by considering a polytope of systems of 8 vertices instead of 4 .

\begin{tabular}{|c|c|}
\hline \multirow[t]{2}{*}{ System } & $\begin{array}{c}\widetilde{G}(s)=\frac{n_{2} s^{2}+n_{0}}{2 s^{2}+d_{1} s+1} \\
n_{0} \in\left[1.25-0.25 \tau_{0}, 1.25+0.25 \tau_{0}\right], n_{2} \in\left[1.25-0.25 \tau_{2}, 1.25+0.25 \tau_{2}\right] \\
d_{1} \in\left[-\tau_{1}, \tau_{1}\right] \text { with } \tau_{i,(i=0, \ldots, 2)} \in\left[0, \tau_{\max }\right]\end{array}$ \\
\hline & $\begin{array}{l}\text { From } \widetilde{G} \text { is deduced a polytope of plants } \Omega_{G} \text { defined by the set of its vertices } \\
G_{i},(i=1 \ldots 8) \text { associated with the } 8 \text { combinations of the bounds of the uncer- } \\
\text { tainty intervals. }\end{array}$ \\
\hline Controller 1 & $\begin{array}{l}\text { A stabilizing controller } C^{e} \text { allowing a maximum uncertainty } \tau_{\max }=1 \\
\qquad C^{e}(s)=\frac{81 s}{1.85 s^{2}+8}\end{array}$ \\
\hline \multicolumn{2}{|c|}{$\begin{array}{l}\text { The roots of the even part of the } 8 \text { characteristic polynomials are } a_{i}= \\
\{-4.3243,-0.5\} \text {. }\end{array}$} \\
\hline Controller 2 & $\begin{array}{l}\text { A stabilizing controller } C^{e} \text { allowing a maximum uncertainty } \tau_{\max }=1.5 \\
\qquad C^{e}(s)=\frac{13.4277 s^{3}+1.1379 s}{0.28 s^{4}+0.65 s^{2}+0.05}\end{array}$ \\
\hline \multicolumn{2}{|c|}{$\begin{array}{l}\text { The roots of the even part of the } 8 \text { characteristic polynomials are } a_{i}= \\
\{-2.2418,-0.5,-0.0797\} \text {. }\end{array}$} \\
\hline
\end{tabular}




\subsubsection{Example 2}

A 3rd order unstable system is considered with four uncertain coefficients.

\begin{tabular}{|c|c|}
\hline System & \multicolumn{1}{c|}{$\widetilde{G}(s)=\frac{s^{3}+n_{2} s^{2}+n_{0}}{d_{3} s^{3}-2 s^{2}+d_{1} s+1}$} \\
& $\begin{array}{l}n_{0} \in\left[1-0.5 \tau_{0}, 1+0.5 \tau_{0}\right], n_{2} \in\left[3-0.5 \tau_{2}, 3+0.5 \tau_{2}\right] \\
d_{1} \in\left[-\tau_{1}, \tau_{1}\right], d_{3} \in\left[1-0.5 \tau_{3}, 1+0.5 \tau_{3}\right] \text { with } \tau_{i,(i=0, \ldots, 3)} \in\left[0, \tau_{\text {max }}\right]\end{array}$ \\
\cline { 2 - 3 } & $\begin{array}{l}\text { From } \widetilde{G} \text { is deduced a polytope of plants } \Omega_{G} \text { defined by the set of its vertices } \\
G_{i},(i=1 \ldots 16) \text { associated with the } 16 \text { combinations of the bounds of the un- } \\
\text { certainty intervals. }\end{array}$ \\
\hline Controller & $\begin{array}{l}\text { A stabilizing controller } C^{e} \text { allowing a maximum uncertainty } \tau_{\text {max }}=1 \\
\text { The roots of the even part of the } 16 \text { characteristic polynomials are } a_{i}=\end{array}$ \\
$\{-1.7,-0.1,-0.01\}$
\end{tabular}

\subsubsection{Example 3}

A 4th order unstable system is treated with five uncertain coefficients.

\begin{tabular}{|c|c|}
\hline \multirow[t]{2}{*}{ System } & $\begin{array}{c}\widetilde{G}(s)=\frac{n_{4} s^{4}+s^{3}+n_{2} s^{2}+n_{0}}{0.1 s^{4}+d_{3} s^{3}-2 s^{2}+d_{1} s+1} \\
n_{0} \in\left[0.75-0.5 \tau_{0}, 0.75+0.5 \tau_{0}\right], n_{2} \in\left[2.25-0.5 \tau_{2}, 2.25+0.5 \tau_{2}\right] \\
n_{4} \in\left[0.11-0.1 \tau_{4}, 0.11+0.1 \tau_{4}\right], d_{1} \in\left[-\tau_{1}, \tau_{1}\right], d_{3} \in\left[1.5-\tau_{3}, 1.5+\tau_{3}\right] \text { with } \\
\tau_{i,(i=0, \ldots, 4)} \in\left[0, \tau_{\max }\right]\end{array}$ \\
\hline & $\begin{array}{l}\text { From } \widetilde{G} \text { is deduced a polytope of plants } \Omega_{G} \text { defined by the set of its vertices } \\
G_{i},(i=1 \ldots 32) \text { associated with the } 32 \text { combinations of the bounds of the un- } \\
\text { certainty intervals. }\end{array}$ \\
\hline Controller & $\begin{array}{l}\text { A stabilizing controller } C^{e} \text { allowing a maximum uncertainty } \tau_{\max }=0.5 \\
\qquad C^{e}(s)=\frac{29.23 s^{3}+395.65 s}{10 s^{4}+138.7 s^{2}+10.5}\end{array}$ \\
\hline & $\begin{array}{l}\text { ts of the even part of the } 32 \text { char } \\
7,-1,-0.1\}\end{array}$ \\
\hline
\end{tabular}




\subsubsection{Example 4}

This is a 5th order unstable system with four uncertain coefficients.

\begin{tabular}{|c|c|}
\hline System & \multicolumn{1}{c|}{$\widetilde{G}(s)=\frac{6 s^{3}+n_{2} s^{2}+22 s+n_{0}}{-0.2 s^{5}-3 s^{4}+d_{3} s^{3}+22 s^{2}+d_{1} s-0.5}$} \\
& $\begin{array}{l}n_{0} \in\left[-3-\tau_{0},-3+\tau_{0}\right], n_{2} \in\left[3-\tau_{2}, 3+\tau_{2}\right] \\
d_{1} \in\left[-11-\tau_{1},-11+\tau_{1}\right], d_{3} \in\left[-16-\tau_{3},-16+\tau_{3}\right] \text { with } \tau_{i,(i=0, \ldots, 3)} \in\left[0, \tau_{\text {max }}\right]\end{array}$ \\
& $\begin{array}{l}\text { From } \widetilde{G} \text { is deduced a polytope of plants } \Omega_{G} \text { defined by the set of its vertices } \\
G_{i},(i=1 \ldots 16) \text { associated with the } 16 \text { combinations of the bounds of the un- } \\
\text { certainty intervals. }\end{array}$ \\
\hline Controller & $\begin{array}{l}\text { A stabilizing controller } C^{e} \text { allowing a maximum uncertainty } \tau_{\text {max }}=1.7 \\
C^{e}(s)=\frac{50.612 s^{3}+65.161 s}{-4.6063 s^{4}-30.132 s^{2}-40.469}\end{array}$ \\
\hline $\begin{array}{l}\text { The roots of the even part of the } 16 \text { characteristic polynomials are } a_{i}= \\
\{-17.275,-3.1836,-0.68588,-0.038819\}\end{array}$
\end{tabular}

\subsubsection{Example 5}

This is a 5th order unstable system with five uncertain coefficients.

\begin{tabular}{|c|c|}
\hline \multirow[t]{2}{*}{ System } & $\begin{array}{c}\widetilde{G}(s)=\frac{-2.5 s^{5}+n_{4} s^{4}-11 s^{3}+n_{2} s^{2}+10 s+n_{0}}{-0.2 s^{5}-31 s^{4}+d_{3} s^{3}-81 s^{2}+d_{1} s-2} \\
n_{0} \in\left[30.5-2.5 \tau_{0}, 30.5+2.5 \tau_{0}\right], n_{2} \in\left[24-2 \tau_{2}, 24+2 \tau_{2}\right] \\
n_{4} \in\left[2.15-0.85 \tau_{4}, 2.15+0.85 \tau_{4}\right], d_{1} \in\left[-72.5-4.5 \tau_{1},-72.5+4.5 \tau_{1}\right] \\
d_{3} \in\left[-4.1-1.4 \tau_{3},-4.1+1.4 \tau_{3}\right] \text { with } \tau_{i,(i=0, \ldots, 4)} \in\left[0, \tau_{\max }\right]\end{array}$ \\
\hline & $\begin{array}{l}\text { From } \widetilde{G} \text { is deduced a polytope of plants } \Omega_{G} \text { defined by the set of its vertices } \\
G_{i},(i=1 \ldots 32) \text { associated with the } 32 \text { combinations of the bounds of the un- } \\
\text { certainty intervals. }\end{array}$ \\
\hline Controller & $\begin{array}{l}\text { A stabilizing controller } C^{e} \text { allowing a maximum uncertainty } \tau_{\max }=1 \\
\qquad C^{e}(s)=\frac{50.612 s^{5}+65.161 s^{3}-53.913 s}{-4.6063 s^{6}-30.132 s^{4}-40.469 s^{2}-28.229}\end{array}$ \\
\hline & $5.32,-1.57,-0.42,-0.034\}$ \\
\hline
\end{tabular}




\subsubsection{Comparison with the robust stabilization approach given in [32] and [33].}

Well known $H_{\infty}$ methods [32] and [33] are used to establish robust stabilizability conditions for a family of uncertain plants and also to synthesize controllers that would stabilize the whole family. These regulators synthesis techniques can be implemented on the examples 1 to 5 , in order to evaluate robustness of our approach in a simple way. For that, an interval system is associated to a family of systems with a central model $G_{0}$ also called nominal model defined through the average values of parameters of the interval model. The plant transfer function is written with a coprime factorization $G_{0}=T_{0} S_{0}^{-1}$ where $T_{0}, S_{0}$ are normalized such that

$$
T_{0}(s) T_{0}(-s)+S_{0}(s) S_{0}(-s)=1 .
$$

Then a family of plants $G$ is characterized by $H_{\infty}$ bounded perturbations to the numerator $T_{0}$ and denominator $S_{0}$ of the normalized coprime factorization of the nomimal system $G_{0}$ as

$$
\mathfrak{F}_{\mathfrak{G}}=\left\{G: G=T S^{-1}, T=T_{0}+\Delta_{T}, S=S_{0}+\Delta_{S}:\left\|\left[\begin{array}{ll}
\Delta_{S} & \Delta_{T}
\end{array}\right]\right\|_{\infty} \leq \beta, \beta>0\right\}
$$

where $\Delta_{T}, \Delta_{S}$ are stable unknown transfer functions representing the uncertainty.

Then $\mathfrak{F}_{\mathfrak{G}}$ is robustly stabilizable by a single linear time invariant controller $C$ iff (see Lemma 3.1 in [32])

$$
\inf _{C}\left\|\left[\begin{array}{c}
C\left(1-G_{0} C\right)^{-1} S_{0}^{-1} \\
\left(1-G_{0} C\right)^{-1} S_{0}^{-1}
\end{array}\right]\right\|_{\infty} \leq \frac{1}{\beta}
$$

where the infinimum is chosen over all compensators $C$ that stabilizes $G_{0}$.

The problem of robust stabilization of normalized coprime factor plant descriptions with $H_{\infty}$ bounded uncertainty is formulated as

$$
\rho\left(G_{0}, G\right)<\beta_{\max }
$$

where $\beta_{\max }$ denotes a maximum $\beta$ and $\rho\left(G_{0}, G\right)$ is the gap-metric between $G_{0}$ and $G$ as defined in [34] and [35] with

$$
\rho\left(G_{0}, G\right)=\max \left(\inf _{Q \in H_{\infty}}\left\|\left[\begin{array}{c}
S_{0} \\
T_{0}
\end{array}\right]-\left[\begin{array}{c}
S \\
T
\end{array}\right] Q\right\|_{\infty}, \inf _{Q \in H_{\infty}}\left\|\left[\begin{array}{c}
S \\
T
\end{array}\right]-\left[\begin{array}{c}
S_{0} \\
T_{0}
\end{array}\right] Q\right\|_{\infty}\right)
$$

The issue of finding $\beta_{\max }$ can be solved via standard $H_{\infty}$ optimization techniques (see [32], [33] and [34]). So, as for the gap metric, any plant $G$ at a distance less than $\beta$ from $G_{0}\left(\beta<\beta_{\max }\right)$ will be stabilized by any compensator $C$ stabilizing the nominal system with a stability margin of $\beta$.

In the case of our approach, an interval plant is represented by a polytope of systems $\Omega_{G}$ defined by its vertices $G_{i}(i=1, \ldots, n)$. It is thus possible to measure for each vertex of the polytope the "distance" to the central plant $G_{0}$ by the gap metric. Denote by $\rho_{m}=\min _{i=1, \ldots, n} \rho\left(G_{0}, G_{i}\right)$ and $\rho_{M}=\max _{i=1, \ldots, n} \rho\left(G_{0}, G_{i}\right)$, the maximum and the minimum of the gap-metrics between $G_{0}$ and $G_{i}(i=1, \ldots, n)$.

Consequently, for each interval plant, the radius of the maximal gap ball $\beta_{\max }$ around the central plant $G_{0}$ associated with the family $\mathfrak{F}_{\mathfrak{G}}$, may be compared to the distance measured by the gap metric between $G_{0}$ and the vertices $G_{i}(i=1, \ldots, n)$ associated with the polytope $\Omega_{G}$. This comparison between the $H_{\infty}$ method given in [32] and [33] and our approach is presented for the examples 1 to 5 in table 1 hereafter where 
- $n$ is the number of vertices $G_{i}(i=1, \ldots, n)$ associated with the polytope of plants $\Omega_{G}$,

- $n_{\rho}$ is the number of vertices $G_{i}(i=1, \ldots, n)$ satisfying $\left.\rho\left(G_{0}, G_{i}\right)\right) \leq \beta_{\max }$.

\begin{tabular}{|c|c|c|c|c|c|c|}
\hline Section & $G_{0}(s)$ & $\beta_{\max }$ & $n$ & $\rho_{m}$ & $\rho_{M}$ & $n_{\rho}$ \\
\hline 5.2 .1 & $\frac{1.25 s^{2}+1.25}{2 s^{2}+1}$ with $\tau_{\max }=1$ & 0.60964 & 8 & 0.6312 & 0.96777 & 0 \\
\hline 5.2 .1 & $\frac{1.25 s^{2}+1.25}{2 s^{2}+1}$ with $\tau_{\max }=1.5$ & 0.60964 & 8 & 0.70467 & 0.999 & 0 \\
\hline 5.2 .2 & $\frac{s^{3}+3 s^{2}+1}{s^{3}-2 s^{2}+1}$ & 0.3329 & 16 & 0.25884 & 0.42977 & 2 \\
\hline 5.2 .3 & $\frac{0.11 s^{4}+s^{3}+2.25 s^{2}+0.75}{0.1 s^{4}+1.5 s^{3}-2 s^{2}+1}$ & 0.24363 & 32 & 0.17906 & 0.2891 & 14 \\
\hline 5.2 .4 & $\frac{6 s^{3}+3 s^{2}+22 s-3}{-0.2 s^{5}-3 s^{4}-16 s^{3}+22 s^{2}-11 s-0.5}$ & 0.1728 & 16 & 0.20878 & 0.79022 & 0 \\
\hline 5.2 .5 & $\frac{-2.5 s^{5}+2.15 s^{4}-11 s^{3}+24 s^{2}+10 s+30.5}{-0.2 s^{5}-31 s^{4}-4.1 s^{3}-81 s^{2}-72.5 s-2}$ & 0.16273 & 32 & 0.034146 & 0.17463 & 31 \\
\hline
\end{tabular}

Table 1: Gap metric results applied to examples 1 to 5

In table 1 , we can observe for every example that $\rho_{M}>\beta_{\max }$. We deduce that the robust stabilization approach developed in [32] and [33] does not allow to take into account an uncertainty level as large as the one considered in the benchmark section 5.2. These results are due to the lost of the uncertainty structure with representation (29). This design limitation of $H_{\infty}$ methods in regard to the uncertainty structure provides conservatism.

Finally, we conclude our numerical benchmark of section 5.2 by pointing out that like in the literature on the stabilization of interval plants, see [18], [19], [20] and [21], our approach is based on sufficient conditions which generate some inevitable conservatism. In other words, if the linear programming problem defined by equation (20) has no solution, it does not mean that there is no robust controller that stabilizes the polytope of plants. In our examples, the limit of stability is reached for a given controller when $\tau_{i,(i=0, \ldots, n)}=\tau_{\max }$ where $n$ is the number of uncertainty coefficient for a given interval plant. It does not imply that there is no robust stabilizing controllers if $\tau_{i,(i=0, \ldots, n)}>\tau_{\max }$. This is illustrated by example 1 where a 2 nd order controller is get with $\tau_{\max }=1$ while a 4 th order controller permits $\tau_{\max }=1.5$. The issue of $\tau_{\max }$ is the one of the compromise between the controller order and the robustness.

\subsection{An example of a polytope of systems not belonging to the family of interval plants}

Consider now a polytope of plants $\Omega_{G}$ defined by the 8 vertices $G_{i}(i=1, \ldots, 8)$ given in table 2 . That is a system whose the polynomial coefficients are not bound by some minimum and maximum values. On the other hand these coefficients can be dependant. The set of all convex combinations 
of vertices $G_{i}(i=1, \ldots, 8)$ forms a convex hull. Note that the systems $G_{i}(i=1, \ldots, 7)$ are unstable plants.

First of all, we show that this polytope $\Omega_{G}$ is not stabilizable by a first order controller. For this purpose, consider the vertex $G_{1}$ of $\Omega_{G}$ of the form (32) with $K=1, \tilde{a}=1$ and $\tilde{b}=1$ and the compensator (33).

\begin{tabular}{|c|c|}
\hline$G_{1}(s)=\frac{1}{s^{3}-s^{2}+s+1}$ & $G_{2}(s)=\frac{-0.24771 s+2.0307}{s^{3}+3 s^{2}-s+6}$ \\
\hline$G_{3}(s)=\frac{-0.059633 s+1.2639}{s^{3}+5}$ & $G_{4}(s)=\frac{-0.31651 s+1.9281}{s^{3}+4 s^{2}+1}$ \\
\hline$G_{5}(s)=\frac{-0.055046 s+1.7033}{s^{3}-s+8}$ & $G_{6}(s)=\frac{0.0091743 s+2.5617}{s^{3}-s^{2}-s+3}$ \\
\hline$G_{7}(s)=\frac{-0.11927 s+2.3815}{s^{3}+s^{2}-s+4}$ & $G_{8}(s)=\frac{-0.44954 s+0.79637}{s^{3}+6 s^{2}+s+2}$ \\
\hline
\end{tabular}

Table 2: Vertices $G_{i}$ of the polytope of plants $\Omega_{G}$

$$
\begin{aligned}
G_{(K, \tilde{a}, \tilde{b})}(s) & =\frac{K}{\tilde{a} s^{3}-\tilde{a} s^{2}+\tilde{a} s+\tilde{b}}, \quad\{K, \tilde{a}, \tilde{b}\} \in \mathbb{R}^{3} \\
C(s) & =\frac{x_{1} s+x_{0}}{y_{1} s+y_{0}}
\end{aligned}
$$

The resulting closed-loop polynomial is

$$
\Phi\left(G_{(K, \tilde{a}, \tilde{b})}, C\right)(s)=\tilde{a} y_{1} s^{4}+\tilde{a}\left(y_{0}-y_{1}\right) s^{3}+\tilde{a}\left(y_{1}-y_{0}\right) s^{2}+\left(\tilde{b} y_{1}+\tilde{a} y_{0}+K x_{1}\right) s+\left(\tilde{b} y_{0}+K x_{0}\right)
$$

Let us remark that this characteristic equation will never be Hurwitz-stable because there are two coefficients of opposite sign, the coefficient of degree 3 and the coefficient of degree 2 . Consequently, the polytope of plants $\Omega_{G}$ can not be stabilized with a first order compensator. Therefore, a controller of higher order must be tested. A second order controller of the following general form

$$
C(s)=\frac{x_{2} s^{2}+x_{1} s+x_{0}}{y_{2} s^{2}+y_{1} s+y_{0}}
$$

is already considered to stabilize this polytope of plants $\Omega_{G}$.

To solve this robust stabilization problem, we desire that the closed-loop characteristic polynomials $\Phi\left(G_{i}, C\right)(i=1, \ldots 8)$ have a same odd part. At this end, we select the two following negative distinct real roots, $b_{1}=-62.203$ and $b_{2}=-2.7973$. Then we find

$$
C(s)=\frac{218 s^{2}+82 s-6}{s^{2}+14 s+78}
$$

With this simultaneous compensator (35), the eight closed-loop characteristic polynomials associated with the eight vertices of $\Omega_{G}$ are Hurwitz-stable and have a same odd part. 


\subsection{Discussion}

The considered numerical examples are not easy to stabilize because simple compensators like constant gain cannot stabilize these uncertain systems. Moreover unstable poles and unstable zeros of these uncertain plants may change which would constitute additional difficulties. It is also useful to observe that in literature this same problem requires the solution of bilinear matrix inequalities (BMIs) which lead to nonconvex optimization problems (see for example [18], [19]) while our approach generates a simple linear programming (LP) problem for which there are efficient solvers with low computational costs. This basic computational procedure founded on linear programming permits to find robust controllers of reasonable order in many cases when the plant order and the number of vertices of the polytope grow. These simulations also show that the order of the compensators is not higher than those used in the usual applications of automatic control: it is at most of the same order that the plant order plus one.

\section{Conclusion}

In this paper, a new approach for stabilizing a polytope of SISO plants has been proposed which yields to a simple controller design for these systems. The Hermite-Biehler theorem and Edge theorem have been the backbones of this framework. An alternative would be to use the HermiteFujiwara criterion, see [36] and [37] instead of the Hermite-Biehler criterion that we apply to stabilize the closed-loop system. So we would have a feedback that would not be based on the common interlacing property but on the properties of the Hermite-Fujiwara matrices. Thus [36] has proved that a polynomial $f$ is Hurwitz-stable iff the Hermite-Fujiwara matrix associated with $f$ is positive definite. Thereafter this property has been extended to define the Hurwitz-stability of a linear combination of several polynomials, see [37]. Assuming that we consider the HermiteFujiwara criterion instead of the Hermite-Biehler criterion, our approach would remain unchanged until theorem 2. Then the conditions (15) of lemma 6 would be modified in order to rewrite the closed-loop characteristic polynomials of the vertices of the polytope with the Hermite-Fujiwara matrices. In this situation, we would get bilinear matrix inequalities (BMIs) conditions which would give a non-convex optimization problem. From the literature, we know that the problem of checking the solvability of a BMI system is NP-hard, see [38]. It is the reason why, the BMIs are converted to linear matrix inequalities (LMIs) with a constraint rank. However this alteration in rank-one LMI optimization problem produces an heuristic algorithm without guaranteeing to find solutions when they exist. For example, in the case of the 2nd order system associated with a polytope of plants of 8 vertices studied in section 5.2, no solution has been found with the Yalmip and SeDuMi solvers for a fourth-order controller by using the Hermite-Fujiwara's criterion. Moreover, this approach generates a higher numerical complexity than the one developed with the Hermite-Biehler criterion. This complexity is linked to the size and to the number of Hermite-Fujiwara matrices. For example, the second order system regulated with a fourth-order controller given in the section 5.2 involves to calculate two hundred Hermite-Fujiwara's matrices.

To summarize, use of the Hermite-Biehler criterion has reduced the stabilization problem of a polytope of systems to a linear programming problem for which there exists efficacious solvers. Our approach has been illustrated by examples showing that the controllers have a reasonable order in spite of the numbers of vertices of the polytope of plants to stabilize and the uncertainty regarded. 
Note that these results could be extended in several ways. Notably we could study the polytopes of plants with a similar controller design than the one given in this paper but in discrete-time or for other stability domains than the unit circle or than the left half-plane of the complex plane. Finally, we could research conditions to stabilize a polytope of systems with a compensator of any order.

\section{Appendix: Stabilization of $\Omega_{G}$ with a same $\Phi\left(G_{i}, C\right)^{o}$}

In the previous sections has been proposed a design of a controller $C$ that guarantees the same even part for the closed-loop characteristic polynomials associated with the vertices of a polytope of systems $\Omega_{G}$. In this appendix are presented similar results but for guaranteeing a same odd part $\Phi\left(G_{i}, C\right)^{o}$ for the closed-loop characteristic polynomials associated with the vertices of $\Omega_{G}$.

Definition 5 (Common odd interlacing property) Consider a finite collection of polynomials $f_{i}^{e}$ and a polynomial $f^{o}$ with their leading coefficients of same sign. Assume that for each pair $\left(f_{i}^{e}, f^{o}\right),(i=1, \ldots, n)$, the roots of $f_{i}^{e}$ and $f^{o}$ are all real, negative simple and distinct defined by the following sets

$$
\begin{aligned}
& \operatorname{roots}\left(f_{i}^{e}\right)=\left\{a_{1}^{i}, \ldots, a_{\ell}^{i}\right\} \\
& \operatorname{roots}\left(f^{o}\right)=\left\{b_{1}, \ldots, b_{\ell}\right\}
\end{aligned}
$$

The polynomials $f_{i}^{e}$ have a common odd interlacing $f^{o}$ iff the $\ell$ roots of each $f_{i}^{e}$ alternate with the $\ell$ roots of $f^{o}$ as below

$$
a_{1}^{i}<b_{1}<a_{2}^{i}<\ldots<a_{\ell}^{i}<b_{\ell}<0
$$

Note that if $f_{i}^{e},(i=1 \ldots, n)$ have a common interlacing $f^{o}$, this implies that for any $j$ th root, we have

$$
\max _{i \in\{1, \ldots, n\}} a_{j}^{i}<b_{j}<\min _{i \in\{1, \ldots, n\}} a_{j+1}^{i} .
$$

Based on this property of common odd interlacing, a corollary is deduced for testing the Hurwitzstability of $f_{i}(s)=f_{i}^{e}\left(s^{2}\right)+s f^{o}\left(s^{2}\right),(i=1 \ldots, n)$.

Corollary 6 (see corollary 1 ) The polynomials $f_{i}(s)=f_{i}^{e}\left(s^{2}\right)+s f^{o}\left(s^{2}\right),(i=1, \ldots, n)$ with $f_{i} \in \mathbb{P}_{m}$ are Hurwitz-stable iff the two following conditions hold.

1. $f^{o}$ has real, simple and negative roots $\left(b_{1}, \ldots, b_{\ell}\right)$.

2. The real coefficients $c_{i, k}(i=1, \ldots, n)$ and $(k=0, \ldots, \ell)$ given by the expansions of $f_{i}^{e}$ $(i=1, \ldots, n)$ as the following sums of products of factors of $f^{o}$

$$
f_{i}^{e}\left(s^{2}\right)=c_{i, 0} f^{o}\left(s^{2}\right)-\sum_{k=1}^{\ell} c_{i, k} \frac{f^{o}\left(s^{2}\right)}{s^{2}-b_{k}} .
$$

are strictly positive. 
From corollary 6 , we can deduce a matrix formulation of $f_{i}^{e}$ and $f^{o}$.

Since $f^{o}\left(s^{2}\right)=\gamma^{o} \prod_{j=1}^{\ell}\left(s^{2}-b_{j}\right)$ with $\gamma^{o} \in \mathbb{R}_{-\{0\}}$ then relation (36) can be simplified as it follows

$$
\begin{aligned}
f_{i}^{e}\left(s^{2}\right) & =\gamma^{o} c_{i, 0} \prod_{j=1}^{\ell}\left(s^{2}-b_{j}\right)-\gamma^{o} \sum_{k=1}^{\ell} c_{i, k} \prod_{\substack{j=1 \\
j \neq k}}^{\ell}\left(s^{2}-b_{j}\right) \\
& =\sum_{k=0}^{\ell} c_{i, k} \sum_{j=0}^{\ell-1} v_{j, k} s^{2 j} \\
& =V A^{o} \Psi_{i}^{o}, \quad i=1, \ldots, n \\
f^{o}\left(s^{2}\right) & =V H^{o}
\end{aligned}
$$

where

$$
\begin{aligned}
\Psi_{i}^{o} & =\left[\begin{array}{lll}
c_{i, 0} & \ldots & c_{i, \ell}
\end{array}\right]^{T} \\
V & =\left[\begin{array}{llll}
1 & s^{2} & \ldots & s^{2 \ell}
\end{array}\right] \\
A^{o} & =\left[\begin{array}{cccc}
v_{0,0} & v_{0,1} & \ldots & v_{0, \ell} \\
v_{1,0} & v_{1,1} & \ldots & v_{1, \ell} \\
\vdots & \vdots & \vdots & \vdots \\
v_{\ell, 0} & v_{\ell, 1} & \ldots & v_{\ell, \ell}
\end{array}\right] \\
H^{o} & =\left[\begin{array}{llll}
h_{0}^{o}, & h_{1}^{o} & \ldots & h_{\ell}^{o}
\end{array}\right]^{T}
\end{aligned}
$$

A reformulation of corollary 6 is now deduced.

Corollary 7 (see corollary 2) The polynomials $f_{i} \in \mathbb{P}_{m}(i=1, \ldots, n)$ are Hurwitz-stable iff the two following conditions hold.

1. $f^{o}$ has real, simple and negative roots $\left(b_{1}, \ldots, b_{\ell}\right)$.

2. There exist $\Psi_{i}^{o} \in \mathbb{R}_{++}^{\ell}$ for $i=1, \ldots, n$, given by (38a).

The Hurwitz-stability issue of a polytope of real polynomials $S_{f}$ defined by (7) may be simplified by considering corollary 3 in [28].

Lemma 8 (see lemma 3) Let $f_{i}$ and $f_{j}$ be in $\mathbb{P}_{m}$, two vertices of $S_{f}$ with their leading coefficients of same sign and with a same odd part $f^{o}$ then the exposed edge $f_{i, j}$ defined by (8) is Hurwitz-stable iff $f_{i}$ and $f_{j}$ are Hurwitz-stable.

Hence, we write.

Corollary 8 (see corollary 3 ) If the vertices $f_{i} \in \mathbb{P}_{m}(i=1, \ldots, n)$, of a polytope of real polynomials $S_{f}$ are Hurwitz-stable with a same odd part $f^{o}$ and if the leading coefficients of $f_{i}$ have a same sign then the polytope of real polynomials $S_{f}$ is Hurwitz-stable.

That implies theorem below. 
Theorem 4 (see theorem 2) Consider a convex polytope of plants $\Omega_{G}$ defined by (9). If there exists a compensator $C$ satisfying the two following relations

$$
\begin{aligned}
& \Phi\left(G_{i}, C\right) \in \mathcal{H}, \\
& \Phi\left(G_{i}, C\right)^{o}=\Phi\left(G_{j}, C\right)^{o}, i=1, \ldots, n-1, j=i+1, \ldots, n .
\end{aligned}
$$

with the leading coefficients of $\Phi\left(G_{i}, C\right)$ of same sign, then $C$ stabilizes $\Omega_{G}$.

Hence the following result by considering $C_{v}$ given by (17) and $\Lambda_{i}^{e}$ and $\Lambda_{i}^{o}$ defined by (18).

Lemma 9 (see lemma 7) Consider a convex polytope of plants $\Omega_{G}$ defined by (9) and $A^{o}, H^{o}$ given by (38c), (38d) respectively. If there exists $C_{v}$ and $\Psi_{i}^{o} \in \mathbb{R}_{++}^{\ell},(i=1, \ldots, n)$ satisfying

$$
\begin{aligned}
\Lambda_{i}^{o} C_{v} & =H^{o}, \quad i=1, \ldots, n, \\
\Lambda_{i}^{e} C_{v} & =A^{o} \Psi_{i}^{o}
\end{aligned}
$$

then $C$ stabilizes $\Omega_{G}$.

Now rewrite relations (40)

$$
B^{o}=\Theta^{o} \chi^{o}
$$

where

$$
\begin{aligned}
& B^{o}=\left[\begin{array}{c}
0 \\
\vdots \\
0 \\
H^{o} \\
H^{o} \\
\vdots \\
H^{o}
\end{array}\right], \quad \Theta^{e}=\left[\begin{array}{ccccc}
A^{o} & 0 & \ldots & 0 & -\Lambda_{1}^{e} \\
\vdots & \vdots & \ddots & \vdots & \vdots \\
0 & 0 & \ddots & A^{o} & -\Lambda_{n}^{e} \\
0 & 0 & \ldots & 0 & \Lambda_{1}^{o} \\
0 & 0 & \ldots & 0 & \Lambda_{2}^{o} \\
\vdots & \vdots & \ldots & \vdots & \vdots \\
0 & 0 & \ldots & 0 & \Lambda_{n}^{o}
\end{array}\right], \\
& \chi^{o}=\left[\begin{array}{ll}
\psi^{o T} & C_{v}^{T}
\end{array}\right]^{T}, \\
& \psi^{o}=\left[\begin{array}{lllll}
\Psi_{1}^{o T} & \Psi_{2}^{o T} & \Psi_{3}^{o T} & \ldots & \Psi_{n}^{o T}
\end{array}\right]^{T} \in \mathbb{R}_{++}^{n(\ell+1)} .
\end{aligned}
$$

There is solutions $\psi^{o}$ and $C_{v}$ to (41) iff [29].

$$
\operatorname{rank}\left(\left[\begin{array}{cc}
B^{o} & \Theta^{o}
\end{array}\right]\right)=\operatorname{rank}\left(\Theta^{o}\right) .
$$

By considering equation (40), the robust stabilization of $\Omega_{G}$ given by lemma 9 is now set as the issue of feasibility of a linear programming problem. Thus, we can deduce the next theorem.

Theorem 5 (see theorem 3) Let $\Omega_{G}$ be a convex polytope of plants defined by (9). Assume that (42) holds then the controller $C$ stabilizes $\Omega_{G}$ if there exists a solution $\chi^{o}$ to the linear programming (LP) problem given by (41). 


\section{References}

[1] Blondel V, Tsitsiklis J. NP-hardness of some linear control design problems. SIAM Journal on Control and Optimization 1997; 35:2118-2127.

[2] Bartlett A, Hollot C, Lin H. Root locations of an entire polytope of polynomials: it suffices to check the edges. Mathematics of Control, Signals, and Systems 1988; 1:61-71.

[3] Vidyasagar M, Viswanadham N. Algebraic design techniques for reliable stabilization. IEEE Transactions on Automatic Control 1982; 27:1085-1095.

[4] Vidyasagar M. Control System Synthesis: A Factorization Approach. MIT Press: Cambridge, USA, 1985.

[5] Youla D, Bongiorno J, Lu C. Single-loop feedback stabilization of linear multivariable dynamical plants. Automatica 1974; 10:159-173.

[6] Ghosh B. Transcendental and interpolation methods in simultaneous system design. Proc. IEEE Conference on Decision \& Control, Fort Lauderdale, USA, 1985.

[7] Ghosh B. Transcendental and interpolation methods in simultaneous stabilization and simultaneous partial pole placement problems. SIAM Journal on Control and Optimization 1986; 24:1091-1109.

[8] Blondel V, Gevers M. The simultaneous stability of three plants is rationally undecidable. Mathematics of Control, Signals, and Systems 1993; 6:135-145.

[9] Abdallah C, Dorato P, Prez F, Docampo D. Controller synthesis for a class of interval plants. Automatica 1995; 31:341-343.

[10] Ghosh B. Some new results on the simultaneous stabilizability of single input, single output systems. Systems \& Control Letters 1985; 6:39-45.

[11] Ghosh B. Simultaneous partial pole-placement: a new approach to multimode system design. IEEE Transactions on Automatic Control 1986; 31:440-443.

[12] Chockalingam G, Dasgupta S. Minimality, stabilizability and strong stabilizability of uncertain plants. IEEE Transactions on Automatic Control 1993; 38:1651-1661.

[13] Chockalingam G, Dasgupta S. Strong stabilizability of systems with multiaffine uncertainties and numerator denominator coupling. IEEE Transactions on Automatic Control 1994; 39:1955-1958.

[14] Fonte C. Conditions for the simultaneous stabilizability of a segment of polynomials. Proc. IEEE American Control Conference, Seattle, USA, 2008.

[15] Chapellat H, Bhattacharyya S. A generalization of the Kharitonov's theorem: robust stability of interval plants. IEEE Transactions on Automatic Control 1989; 39:306-311.

[16] Chapellat H, Bhattacharyya S. An alternative proof of Kharitonov's theorem. IEEE Transactions on Automatic Control 1989; 34:448-450. 
[17] Sideris A. An efficient algorithm for checking the robust stability of a polytope of polynomials. Mathematics of Control, Signals, and Systems 1991; 4:315-337.

[18] Henrion D, Šebek M, Kučera V. Positive polynomials and robust stabilization with fixed-order controllers. IEEE Transactions on Automatic Control 2003; 48:1178-1186.

[19] Henrion D, Arzelier D, Peaucelle D, Šebek M. An LMI condition for robust stability of polynomial matrix polytopes. Automatica 2001; 37:461-468.

[20] Karimi A, Khatibi H, Longchamp R. Robust control of polytopic systems by convex optimization. Automatica 2007; 43:1395-1402.

[21] Khatibi H, Karimi A, Longchamp R. Fixed-order controller design for polytopic systems using LMIs. IEEE Transactions on Automatic Control 2008; 53:428-434.

[22] Bianchini G, Tesi A, Vicino A. Synthesis of robust strictly positive real systems with $l_{2}$ parametric uncertainty. IEEE Transactions on Circuits and Systems-I: Fundamental Theory and Applications 2001; 48:438-450.

[23] Patel V, Datta K. A note on interpolation with a strictly positive real function. IEEE Transactions on Automatic Control 1995; 40:1960-1962.

[24] Patel V, Datta K. Classification of units in $H_{\infty}$ and an alternative proof Kharitonov's theorem. IEEE Transactions on Circuits and Systems-I: Fundamental Theory and Applications 1997; 44:454-458.

[25] Gantmacher F. The Theory of Matrices. Chelsea Publishing Company: New York, 1959.

[26] Fonte C, Delattre C. Conditions for interpolation of stable polynomials. Proc. Mathematical Theory of Networks and Systems Symposium, Budapest, Hungary, 2010.

[27] Fonte C, Meddeb H, Zasadzinski M. An interpolation approach with stable polynomial interpolants applied to the simultaneous stabilization of a segment of systems. Proc. IEEE Conference on Decision 86 Control, Los Angeles, USA, 2014.

[28] Fonte C, Delattre C. Wronskian-based tests for stability of polynomial combinations. Systems \& Control Letters 2011; 60:590-595.

[29] Ben-Israel A, Greville T. Generalized Inverses: Theory and Applications. Wiley: New York, 1974.

[30] Barmish B. New Tools for Robustness of Linear Systems. Macmillan Publishing Company: New York, 1994.

[31] Bhattacharyya S, Chapellat H, Keel L. Robust Control, The Parametric Approach. Prentice Hall: Englewood Cliffs, New Jersey, 1995.

[32] Glover K, McFarlane D. Robust stabilization of normalized coprime factor plant descriptions with $H_{\infty}$-bounded uncertainty. IEEE Transactions on Automatic Control 1989; 34:821-830.

[33] McFarlane D, Glover K. A loop-shaping procedure using $H_{\infty}$ synthesis. IEEE Transactions on Automatic Control 1992; 37:759-769. 
[34] Georgiou T, Smith M. Optimal robustness in the gap metric. IEEE Transactions on Automatic Control 1990; 35:673-686.

[35] Georgiou T. On the computation of the gap metric. Systems $\&$ Control Letters 1988; 11:253257.

[36] Jury E. Inners and stability of dynamic systems. IEEE Transactions on Automatic Control 1982; 27:1085-1095.

[37] Henrion D, Šebek M, Tarbouriech S. Algebraic approach to robust controller design: a geometric interpretation. Proc. IEEE American Control Conference, Philadelphia, USA, 1998.

[38] Toker O, Özbay H. On the NP-hardness of solving bilinear matrix inequalities and simultaneous stabilization with static output feedback. Proc. IEEE American Control Conference, Seattle, USA, 1995. 